\title{
UN NOUVEL APPAREIL D'ÉTUDE TOXICOLOGIQUE UTILISANT DES CARPILLONS
}

\author{
par IV. K. Besch ${ }^{1}$ el H. JuhNKe ${ }^{2}$.
}

Le problème de la délermination de la toxicité des eaux superficielles ou des eaux d'égouts exige l'utilisation de méthodes biologiques. La méthode la plus couramment utilisée est celle du «Fish-test» (poisson-test).

Au cours d'un test, l'absence de dommage pour un poisson ne permet pas de conclure à la possibilité d'une vie prolongée dans l'eau étudiée. A une concentration donnée, les substances toxiques peuvent en effet s'avérer inoffensives pour le poisson-test mais réduire ou même éliminer complètement les organismes nutritifs. De même, le poisson-test ne fournit aucune information sur l'importante question de la dégradation de la matière organique et de l'oxygénation biogénique, c'est-à-dire de l'arrêt de l'autoépuration d'une eau.

Par contre, dans un cours d'eau le Fish-test reste actuellement la seule méthode permettant de détecter un afflux de concentration très élevée en substances toxiques avec précision et rapidité. La mortalité ou même les lésions causées aux poissons sont considérées par le public comme le signal d'alarme de l'intoxication des eaux. De plus, l'utilisation de poissons, mieux que cclle d'invertébrés, permet de tirer des déductions concernant la toxicologie humaine.

Ces raisons ont conduit la Landesstelle für Gewässerkunde du Bade-Wurtemberg (Karlsruhe) à mettre au point une nouvelle méthode de fish-lest permettant le contrôle de la toxicité des eaux résiduaires ou d'importants cours d'eau tel que le Rhin. Des carpillons de l'été (6-10 $\mathrm{mm}$ de longueur) ont été utilisés par suite de facilités d'approvisionnement ainsi que pour leur résislance aux températures élevées.

En général, on considère comme mesure de toxicité une morta-

1. Landesstelle für Gewässerkunde, Baden-Württemberg in Karlsruhe (Allemagne).

2. Landesanstalt für Gewässerkunde Nordrhein-Westfalen in Krefeld (Allemagne). 

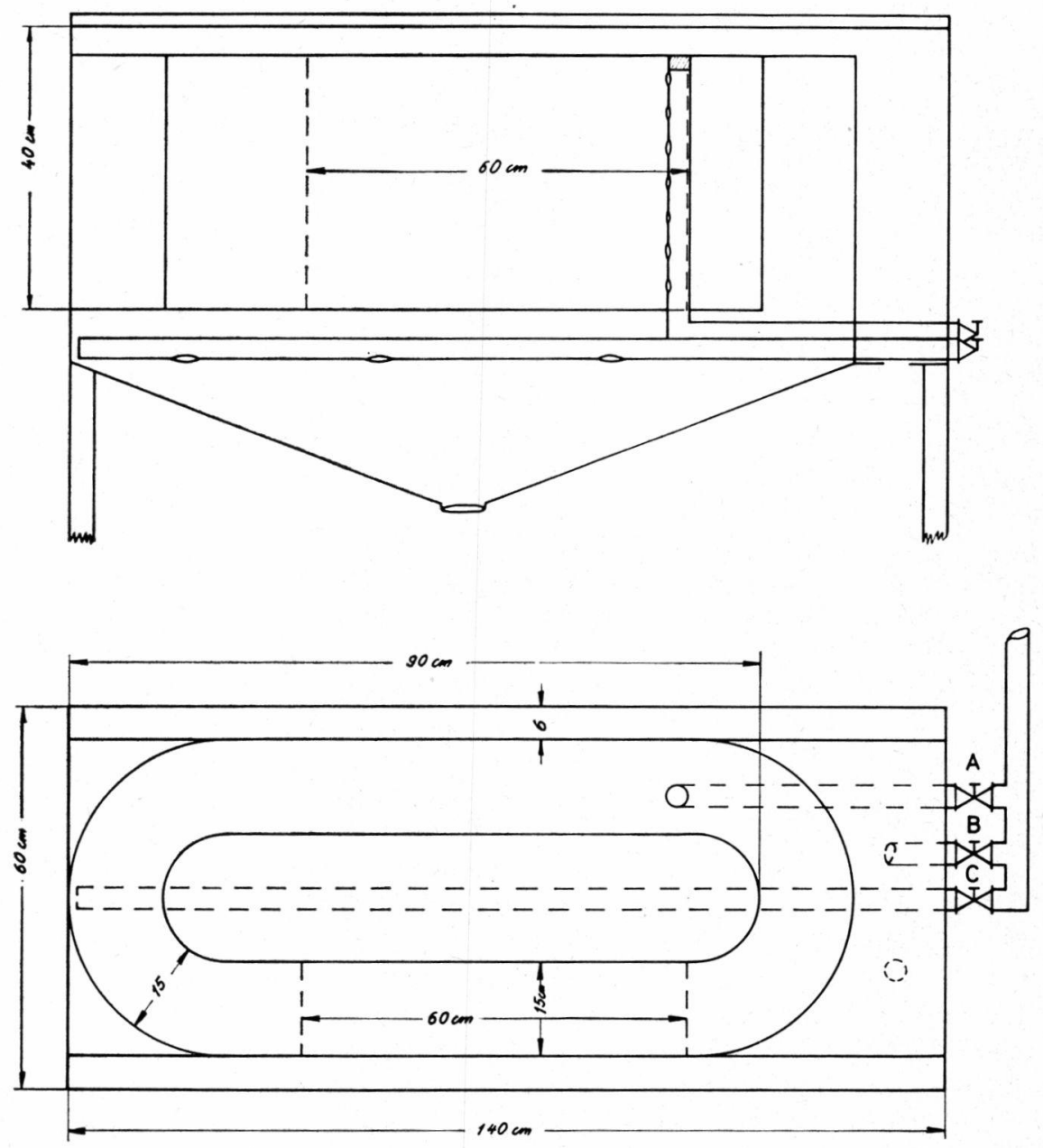

Fig. 1. - Appareillage de tests toxicologiques en eau courante. En haut : vue latérale; en bas : vue de dessus.

lité de $50 \%$ ('Tlm) des animaux. La toxicité est aiguë si ce pourcentage est atteint avant 96 heures; elle est chronique s'il n'apparaît qu'après 96 heures (SPrague 1969).

Les expériences se déroulent habituellement en eau stagnante. Dans des tests de contrôle continu de la toxicité d'un cours d'eau (tests dynamiques), le milieu est constamment renouvelé; les conditions hydrodynamiques offertes aux poissons demeurent néanmoins celles d'une eau stagnante.

Dans nos tests, au contraire, les poissons sont obligés de réagir aux excitations d'un courant défini à la fois en direction et en puissance. 
Selon les propositions de ZaHNER (1962) nous considérons comme mesure de la toxicilé, le temps s'écoulant jusqu’à l'apparition de la manifestation biologique et non jusqu'à la mort.

La manifestation biologique se traduit par l'incapacité pour les poissons de se maintenir dans un courant modéré de vitesse connue.

Cette méthode présente des avantages considérables :

1. - L'estimation de la manifestation - les poissons sont entraînés par le courant contre le grillage de la cage - est beaucoup plus précise que celle du moment de la mort.

2. - Les dispersions statistiques sont plus faibles que celles concernant la mortalité.

3. - La manifestation se produit bien avant la mort, d'où une économie de temps pour la conduite des lests.

L'appareil que nous avons conçu tient compte de deux propositions :

$1^{\circ}$ L'eau à surveiller peut s'écouler uniformément.

$2^{\circ}$ La carpe ne peut se maintenir longtemps dans un courant relativement fort.

Cet appareil est représenté sur la figure 1. Il a été réalisé dans les ateliers de la firme Bechthold (Eggenstein près de Karlsruhe). Il peut être utilisé au laboratoire, en circuit fermé, pour des essais statiques. Mais, l'appareil peut aussi être traversé par de l'eau pompée dans la rivière à surveiller (essais dynamiques).

Alternativement, un courant vertical faible $(0,5 \mathrm{~cm} / \mathrm{s})$ ou horizontal plus fort $(6-15 \mathrm{~cm} / \mathrm{s})$ peut être utilisé.

Un canal, au-dessus de l'appareil, assure l'écoulement en eau. On obtient un courant horizontal par l'ouverture de petits trous disposés le long d'un tube au milieu du canal ovale. On obtient un courant vertical par l'ouverture du robinet $C$ (A étant fermé) ce qui laisse entrer l'eau à travers les perforations dı fond.

Une partie du canal ovale, longue de $60 \mathrm{~cm}$, large de $15 \mathrm{~cm}$ et haute de $40 \mathrm{~cm}$, limitée par deux grillages, sert d'emplacemenl aux poissons.

En courant horizontal, l'eau circule de gauche à droite. La manifestation survient lorsque les carpes commencent à s'appuyer à l'aide de leur nageoire caudale au grillage de droite .

Des cellules photoélectriques permettent d'enregistrer :

1" l'activité des poissons; 


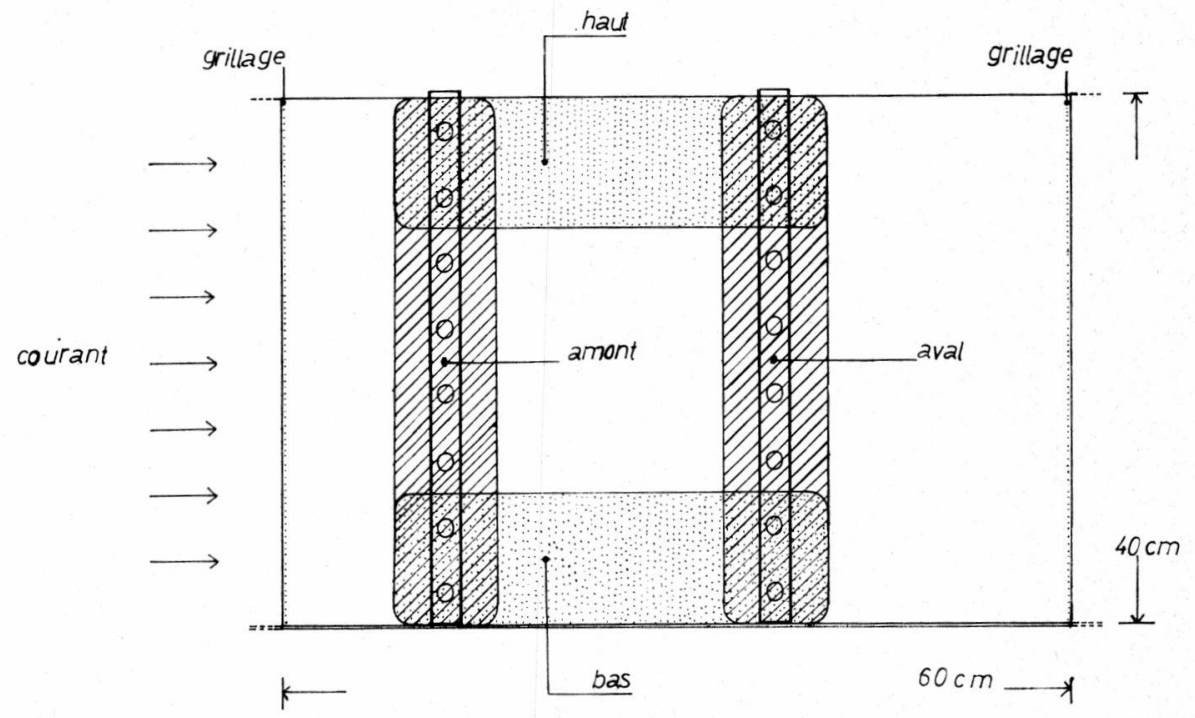

Fig. 2. - Vue latérale schématique de l'enceinte des poissons, avec la disposition des cellules photoélectriques permettant de mesurer l'activité aux différents niveaux.

$2^{\circ}$ la rapidité de leurs déplacements;

$3^{\circ}$ leur localisation dans la cage (à gauche ou à droite, en surface ou près du fond).

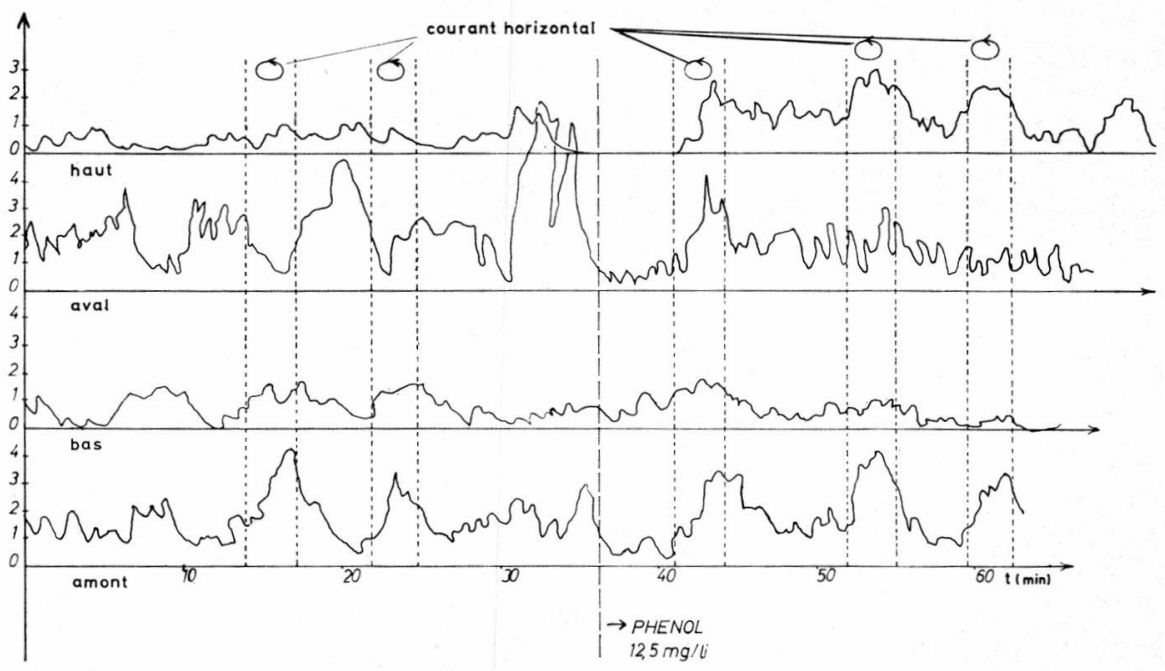

FIG. 3. - Enregistrement de l'activité de 5 carpillons avant et après l'adjonction de phénol dans l'eau; en ordonnée : le nombre de passages par seconde. 
Un appareillage électronique (Firme Karl Heinz Weiss, Karlsruhe, Rheinstrasse) permet d'enregistrer, plutôt que des passages individuels, les passages par unité de temps : 10 secondes (fig. 3 ). Au début, les poissons se trouvent dans l'eau pure (partie gauche de la figure). Puis, du phénol est ajouté progressivement jusqu’à une concentration de $12,5 \mathrm{mg} / 1$ (partie droite).

Après l'adjonction de poison, on observe une période (6 minutes) d'inactivité presque tolale suivie d'une activité accrue près de la surface. Comme l'a déjà signalé $Z_{A H N E R}$ (1962) on observe, bien avant la manifestation de la toxicité, des comportements anormaux variés, différant selon la nature du poison utilisé.

\section{RÉSUMÉ}

L'incapacité des poissons à se maintenir dans un courant d'eau modéré est considérée comme la manifestation biologique de la présence de poison. La toxicité est mesurée par le temps écoulé jusqu'à la manifestation. De jeunes carpes (espèce d'eau stagnante) sont utilisées. I.'appareil permet d'obtenir alternativement un courant vertical $(0,5 \mathrm{~cm} / \mathrm{s})$ ou horizontal $(6-15 \mathrm{~cm} / \mathrm{s})$. L'eau peut circuler en circuit fermé (tests statiques) ou en circuit ouvert (tests dynamiques). Les comportements provoqués par l'effet du poison sur les carpes sont enregistrés au moyen de cellules photoélectriques.

\section{A NEW APPARATUS FOR TOXICOLOGICAL STUDIES WITH CARP}

The inability of experimental fish to swin against a moderate current is used as a sign of the presence of poison. The toxicity is given by the time needed for this to occur.

Yearlings carp (still water species) are used. The apparatus which can produce a very weak vertical current and a strong horizontal one, is described. The water used can either be re-circulated (static experiments) or can flow through the apparatus (dynamic experiments).

It is suggested that the toxic effects on the fish should be registered by photoelectric equippment.

\section{BESCHREIBUNG EINER NEUEN METHODE TOXICOLOGISCHER UNTERSUCHUNGEN MTT KARPFEN}

Als Zeichen von Giftschadwirkungen bei toxikologischen Untersuchungen wird die Manifestation, die Unfähigkeit der Testfische genommen, sich in einer mäßigen gerichteten Strömung zu halten, und als Maß die Zeit, die bis dahin verstreicht.

Um einer Dauerströmung ungewohnten (einsömmrigen) Karpfen auch im Fließwassertest verwenden zu können, wurde eine Apparatur gebaut, in der alternativ eine sehr schwache vertikale und eine mäßige horizontale Strömung eingestellt werden kann. 
Das Wasser kann entweder im Kreislauf herumgeführt werden (statische Tests) oder ständig erneuert werden (dynamische Tests).

Es wird vorgeschlagen auch die Effekte von toxischen Einwirkungen auf das Fischverhalten mittels photoelektrischer Einrichtungen zu registrieren.

\section{TRAVAUX GITÉS}

Alabaster (J. S.). 1967. - Estimating the effect of Herbicides on fish. European Water Research Council Symposium editor Ministry of Agriculture, Fisheries and Food. 3 pp.

Sprague (J.B.). 1969. - Measurement of Pollutant Toxicity to Fish Bioassay Methods for Acute Toxicity - Water Research, 3 : 793821.

Zahner (R.). 1962. - Uber die Wirkung von Teibstoffen und Olen auf Regenbogenforellen. Vom Wasser, 29 : 142-177. 